\title{
Infundibular sparing versus transinfundibular approach to the repair of tetralogy of Fallot
}

\author{
Mary K. Olive MD ${ }^{1}$ (D) | Charles D. Fraser MD ${ }^{2}$ | Shelby Kutty $M^{3}$ | \\ Emmett D. McKenzie MD ${ }^{4}$ | James M. Hammel MD ${ }^{5}$ | Rajesh Krishnamurthy $M^{6}$ | \\ Nicolas A. Dodd BM ${ }^{7}$ | Shiraz A. Maskatia MD ${ }^{8}$ (D)
}

\author{
${ }^{1}$ Department of Pediatrics, Section of \\ Pediatric Cardiology, Congenital Heart \\ Center, C.S. Mott Children's Hospital, \\ University of Michigan, Ann Arbor, Michigan \\ ${ }^{2}$ Department of Surgery and Perioperative \\ Care, Texas Center for Pediatric and \\ Congenital Heart Disease, University of \\ Texas Dell Medical School, Dell Children's \\ Medical Center, Austin, Texas \\ ${ }^{3}$ Department of Pediatrics, Taussig \\ Congenital Heart Center, Johns Hopkins \\ University, Baltimore, Maryland \\ ${ }^{4}$ Section of Congenital Heart Surgery, \\ Texas Children's Hospital, Baylor College of \\ Medicine, Houston, Texas \\ ${ }^{5}$ Section of Cardiovascular Surgery, \\ University of Nebraska College of Medicine, \\ Omaha, Nebraska \\ ${ }^{6}$ Section of Diagnostic Radiology, \\ Nationwide Children's Hospital, Ohio State \\ University, Columbus, Ohio \\ ${ }^{7}$ Section of Pediatric Radiology, Texas \\ Children's Hospital, Baylor College of \\ Medicine, Houston, Texas \\ ${ }^{8}$ Section of Pediatric Cardiology, Stanford \\ University, Palo Alto, California
}

\section{Correspondence}

Mary K. Olive, MD, Department of Pediatrics, Section of Pediatric Cardiology, Congenital Heart Center, C.S. Mott Children's Hospital, University of Michigan, 1540 East Medical Center Drive, Floor 11, Room 715-Z, Ann Arbor, MI 48109.

Email:mkolive@med.umich.edu

\begin{abstract}
Introduction: The right ventricular infundibular sparing approach (RVIS) to the repair of tetralogy of Fallot (TOF) avoids a full-thickness ventricular incision, typically utilized in the transinfundibular ( $\mathrm{TI}$ ) method.

Methods: We performed a retrospective, age-matched cohort study of patients who underwent RVIS at Texas Children's Hospital or TI at Children's Hospital Medical Center in Nebraska and subsequently underwent cardiac magnetic resonance imaging (CMR). We compared right ventricular end-diastolic and systolic volumes indexed to body surface area (RVEDVi and RVESVi) and right ventricular ejection fraction (RVEF) as primary endpoints. Secondary endpoints were indexed left ventricular diastolic and systolic volume (LVEDVi and LVESVi), left ventricular ejection fraction (LVEF), right ventricular (RV) sinus ejection fraction (EF) and RV outflow tract EF (RVOT EF).

Results: Seventy-nine patients were included in the analysis; 40 underwent RVIS and 39 underwent TI repair. None of the patients in the TI repair group had an initial palliation with a systemic to pulmonary arterial shunt compared to seven (18\%) in the RVIS group $(P<.01)$. There was no appreciable difference in RVEDVi $(122 \pm 29 \mathrm{cc} /$ $\mathrm{m}^{2}$ vs $\left.130 \pm 29 \mathrm{cc} / \mathrm{m}^{2}, P=.59\right)$ or pulmonary regurgitant fraction ( $40 \pm 13$ vs $37 \pm 18$, $P=.29$ ) between the RVIS and TI groups. Compared to the TI group, the RVIS group had higher RVEF $(54 \pm 6 \%$ vs $44 \pm 9 \%, P<.01)$, lower RVESV $\left(57 \pm 17 \mathrm{cc} / \mathrm{m}^{2}\right.$ vs $67 \pm 25 \mathrm{cc} / \mathrm{m}^{2}, P=.03$ ), higher LVEF (61 $\pm 11 \%$ vs $54 \pm 8 \%, P<.01$ ), higher RVOT EF $(47 \pm 12 \%$ vs $41 \pm 11 \%, P=.03)$, and higher RV sinus EF ( $56 \pm 5 \%$ vs $49 \pm 6 \%, P<.01)$ Conclusions: In this selected cohort, patients who underwent RVIS repair for TOF had higher right and left ventricular ejection fraction compared to those who underwent TI repair.
\end{abstract}

\section{KEYWORDS}

cardiac magnetic resonance imaging, right ventricular infundibular sparing repair, tetralogy of Fallot

\section{1 | INTRODUCTION}

Childhood surgical correction of tetralogy of Fallot (TOF) results in low short-term mortality and morbidity. ${ }^{1,2}$ right ventricular (RV) dysfunction is associated with long-term morbidities, and related to chronic pulmonary regurgitation, RV fibrosis and RV obstruction, obesity, and gender. ${ }^{3-8}$ Infundibular scarring has been associated with worsening RV dilation and dysfunction in an animal model. ${ }^{9}$ 
The optimal surgical strategy to the repair of TOF is an area of controversy. Many institutions perform early elective repair. ${ }^{10,11}$ This transinfundibular ( $\mathrm{TI}$ ) approach relieves RV obstruction and cyanosis early, but requires a full-thickness incision in the infundibulum of the RV. ${ }^{12}$ In contrast, the right ventricular infundibular sparing technique (RVIS) avoids a full-thickness infundibular incision (Figure 1). The proposed benefit of this strategy is preservation of infundibular contractility. ${ }^{13}$ The aim of the present study was to compare RV size and function by cardiac magnetic resonance imaging (CMR) in patients who underwent RVIS repair to those who underwent $\mathrm{TI}$ approach.

\section{2 | METHODS}

We performed an age-matched; multi-institutional retrospective cohort study comparing CMR measurements of RV size and function in patients with TOF repaired via RVIS at Texas Children's Hospital (TCH) or TI at Children's Hospital and Medical Center, University of Nebraska (CHMC). Pediatric (<18 years of age) patients with repaired TOF who underwent CMR evaluation prior to any repeat surgical intervention between January 2004 and April 2014 were identified. Right ventricular infundibular sparing technique approach was defined by avoidance of a full-thickness infundibular incision and transatrial, transpulmonary approach to ventricular septal defect closure and resection of infundibular muscle. ${ }^{11,12}$ When the pulmonary annulus was inadequate, the pulmonary arteriotomy extended across the annulus by $\leq 5 \mathrm{~mm}$. Repair via the RVIS approach is typically performed at 6-8 months of age. An intervening systemic to pulmonary artery shunt is necessary prior to complete repair in approximately $17 \%$, and a transannular patch in approximately $75 \%$ of cases. ${ }^{12}$ Three patients underwent $\mathrm{TI}$ at $\mathrm{TCH}$ due to the desire to avoid shunt physiology due to the presence of concomitant lung disease (extreme prematurity, congenital diaphragmatic hernia, and/or multiple respiratory infections), and were excluded from the study. The Tl approach was defined by a full-thickness incision of the infundibulum. In cases with significant infundibular hypoplasia, a TIpatch was utilized to relieve obstruction. Otherwise, infundibulotomies were closed primarily. Throughout the study period, ventriculotomies were

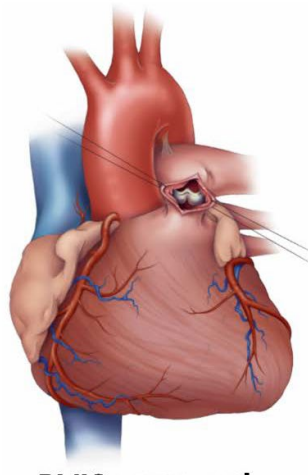

RVIS approach

\section{Transinfundibular Approach}

FIGURE 1 Comparison of the right ventricular infundibular sparing repair (left) and the transinfundibular repair (right) typically performed via a vertical incision. Patients in both RVIS and TI groups were excluded if they had pulmonary atresia or if they received an RV to pulmonary artery conduit during initial repair. Patients were categorized based on age at the time of CMR as follows: 3-5 years, 5-9 years, 9-13 years, and 13-18 years. To control for the effect of age on RV size and function, patients were matched 1:1 within these age categories. When the number of TI versus RVIS patients in each age category were disparate, patients were selected for inclusion with the use of a random number generator.

CMR studies were performed using a 1.5-T whole body scanner using a cardiac or thoracic coil and previously published protocols. ${ }^{14}$ ECG-gated, breath-held, or free-breathing cine imaging was performed in the short-axis plane using balanced steady-state free precession sequences. The stated indications for CMR in each patient were to evaluate biventricular size and systolic function and to evaluate pulmonary regurgitant fraction in the context of repaired TOF. Referrals for CMR were dependent upon the individual practitioners at each institution. Generally speaking, patients were referred as part of the standard assessment of a patient with repaired TOF. CMR studies from both institutions were anonymized and loaded onto an independent workstation (CMR 42, Circle CVI Inc., Calgary, Canada). Measurements were performed by a radiology technician with expertise in postprocessing CMR studies in patients with congenital heart disease (NAD) and reviewed by a pediatric cardiologist with expertise in CMR (SAM). Both observers were blinded to surgical technique, institution, and clinical outcome. The RV was traced to the level of the pulmonary valve annulus, and trabeculations were included in cavity size. The RV was divided into the RV sinus and the RV outflow tract (RVOT) in both diastole and systole. The RVOT/MPA border was defined by the pulmonary valve annulus. The border RVOT/RV sinus border was defined by the septal and parietal bands, similar to prior studies.15-17 RV sinus and RVOT ejection fractions (EF) were calculated. Phase contrast imaging was employed to calculate pulmonary regurgitant fraction (PRF). ${ }^{18}$ The main pulmonary artery is often significantly dilated in the setting of repaired TOF. As peak velocity in the main pulmonary artery is underestimated by cross-sectional phase contrast imaging in this setting, this value was not recorded. In-plane phase contrast imaging of the RVOT obtained in a minority of the studies, and therefore was not collected as part of this study.

Primary endpoints were: RVEF, indexed RV end-diastolic volume (RVEDVi), and indexed RV end-systolic volume (RVESVi). RV systolic dysfunction was defined as $E F<45 \%$, as this is a previously identified risk factor for major adverse events. ${ }^{4,19}$ Data were expressed as mean \pm standard deviation when parametric and median and interquartile range (IQR) when non-parametric. Shapiro-Wilk test was applied to determine normality. Statistical analyses were carried out using Statistical Package for Social Sciences (SPSS IBM, Chicago IL, Version 23). Categorical variables were analyzed using chi-square test. Continuous variables were compared using Student's ttest (parametric) or Mann-Whitney $U$ test (nonparametric). Stepwise multivariable linear regression was used to evaluate the primary outcome measures of RVEF, RVEDVi, and RVESVi using the following independent variables: RVIS repair, transannular patch, age at CMR, 
and gender. Independent variables were removed from the regression analysis in a stepwise fashion for $P$ values greater than .15 . The assumptions of linearity, homoscedasticity, independence of errors, and unusual points and normality of residuals were met. There was independence of residuals, as assessed by a Durbin-Watson statistic of 2.08. As later age at repair and the possible use of an intervening systemic to pulmonary artery shunt are inherent characteristics of the RVIS approach, these variables were not controlled for in the model.

This project was supported by the cardiovascular research institute at $\mathrm{TCH}$. Approval was obtained from the Internal Review Boards at the Baylor College of Medicine and University of Nebraska College of Medicine. Consent was waived.

\section{3 | RESULTS}

Patients underwent TOF repair between February 1994 and May 2011. Information on surgical volume was not available from either center prior to 1995. From 1995-2011, the total number of cardiopulmonary bypass cases and TOF repairs performed were 7384 and 491 (TCH) and 2669 and 134 (CHMC). At both institutions, surgical volume increased steadily across time. Surgical mortality rates following repair of TOF were 1.2\% $(\mathrm{TCH})$ and $2.0 \%(\mathrm{CHMC})$. No patients who received an intervening systemic to pulmonary artery shunt died prior to TOF repair. A total of 112 patients who met inclusion criteria underwent CMR at either TCH $(N=67,14 \%)$ or CHMC $(N=45,34 \%)$ during the study period. Patients who underwent TOF repair and were followed clinically at other institutions were not included. After age-matching, there were 40 patients in each cohort. After patient selection had concluded and data analysis had begun, one subject was identified in the TI group was excluded due to insufficient CMR images for analysis. Table 1 displays baseline demographic and surgical data. The median age at TOF repair was significantly younger in the TI group. None of the patients in the TI group required a staged surgical approach. Median age at CMR was not different between the two groups (Table 2).

Average RVEF was higher in the RVIS group compared to the TI group (55 $\pm 5 \%$ vs $48 \pm 6 \%, P \leq .01$ ). The odds of RV dysfunction (RVEF < 45\%) were $8.5 \times(1.7-48)$ higher in the TI group compared to the RVIS group. RV outflow tract EF and RV sinus EF were higher in the RVIS group compared to the TI group ( $47 \pm 12 \%$ vs $40 \pm 12 \%$, $P=.01$ and $56 \pm 5 \%$ vs $49 \pm 6 \%, P<.01$, respectively, Figure 2$)$. LVEF was higher in the RVIS group compared to the TI group, $59 \pm 4 \%$ compared to $56 \pm 8 \%(P=.04)$. There was a modest correlation between LVEF and RVEF, with $R=.56, P<.01$ (Figure 3). Figure 4 displays average RVEF for both groups within each age category. Right ventricular end-diastolic and systolic volumes index was significantly lower in the RVIS group compared to the TI group $(P=.02)$, while RVEDVi, and LVEDVi were not significantly different between the two groups (Figure 5 and Table 3 ).

On univariable analysis, within the RVIS cohort, there was slightly lower RVEF in males compared to females $(53 \pm 4 \%$ vs $56 \pm 4 \%$,
TABLE 1 Demographic and surgical data

\begin{tabular}{|c|c|c|c|}
\hline & Transinfundibular & RVIS & \multirow[b]{2}{*}{$P$ value } \\
\hline & $N=39$ & $N=40$ & \\
\hline Male & 27 (69\%) & 24 (60\%) & .40 \\
\hline Trisomy 21 & $2(5 \%)$ & $1(3 \%)$ & .54 \\
\hline $\begin{array}{l}\text { 22q11 deletion } \\
\text { syndrome }\end{array}$ & $3(8 \%)$ & $3(8 \%)$ & .98 \\
\hline $\begin{array}{l}\text { Median (IQR) age at } \\
\text { repair in months }\end{array}$ & $4.0(2.5-5.8)$ & $9.8(6.2-14.8)$ & $<.01$ \\
\hline $\begin{array}{l}\text { Systemic to pulmonary } \\
\text { artery shunt }\end{array}$ & 0 & $7(18 \%)$ & $<.01$ \\
\hline Transannular patch & 29 (74\%) & 35 (88\%) & .14 \\
\hline
\end{tabular}

Abbreviation: RVIS, right ventricular infundibular sparing approach.

TABLE 2 Age distribution

\begin{tabular}{|c|c|c|c|}
\hline & Transinfundibular & RVIS & \\
\hline & $N=39$ & $N=40$ & $P$ value \\
\hline $\begin{array}{l}\text { Median (IQR) age } \\
\text { at CMR in years }\end{array}$ & $11.5(4.4-15)$ & $9.7(6.0-14)$ & .65 \\
\hline $\begin{array}{l}\text { Age category } 1 \\
\text { ( }<5 \text { years) }\end{array}$ & 8 & 8 & \\
\hline $\begin{array}{l}\text { Age category } 2 \\
\text { (5-9 years) }\end{array}$ & 7 & 8 & \\
\hline $\begin{array}{l}\text { Age category } 3 \\
\text { (9-13 years) }\end{array}$ & 9 & 9 & \\
\hline $\begin{array}{l}\text { Age category } 4 \\
\text { (13-18 years) }\end{array}$ & 15 & 15 & \\
\hline
\end{tabular}

Abbreviations: CMR, cardiac magnetic resonance imaging; RVIS, right ventricular infundibular sparing approach.

$P=.04)$. Within the TI cohort, there was no significant difference in RVEF between males and females ( $47 \pm 6 \%$ vs $49 \pm 7 \%, P=.63$ ). Within the RVIS cohort, RVEDVi in males was $136 \pm 32 \mathrm{~mL} / \mathrm{m}^{2}$ compared to $122 \pm 23 \mathrm{~mL} / \mathrm{m}^{2}$ in females $(P=.15)$, and within the TI cohort the average RVEDVi in males was $148 \pm 45 \mathrm{~mL} / \mathrm{m}^{2}$ compared to $120 \pm 41 \mathrm{~mL} / \mathrm{m}^{2}$ in females $(P=.07)$. The presence of a transannular incision did not have a significant association with global or segmental ventricular size or function (Table 4). Patients who underwent transannular incision had higher PRF compared to those who did not. Within the RVIS cohort, older age at CMR correlated with higher $\operatorname{RVESVi}(R=.5, P=.001)$ and RVEDVi $(R=.6, P<.01)$. There was no appreciable association between age and RVEF or LVEF in the RVIS group; we found no appreciable association between age and any outcome variable within the TI group. Within the RVIS group, there was no significant difference in RVEF, RVEDVi, RVESVi, RVOT EF, or RV body EF between patients who did or did not receive a systemic to pulmonary artery shunt. Within each cohort, there were no significant associations between age at TOF repair and any of the outcome variables. 


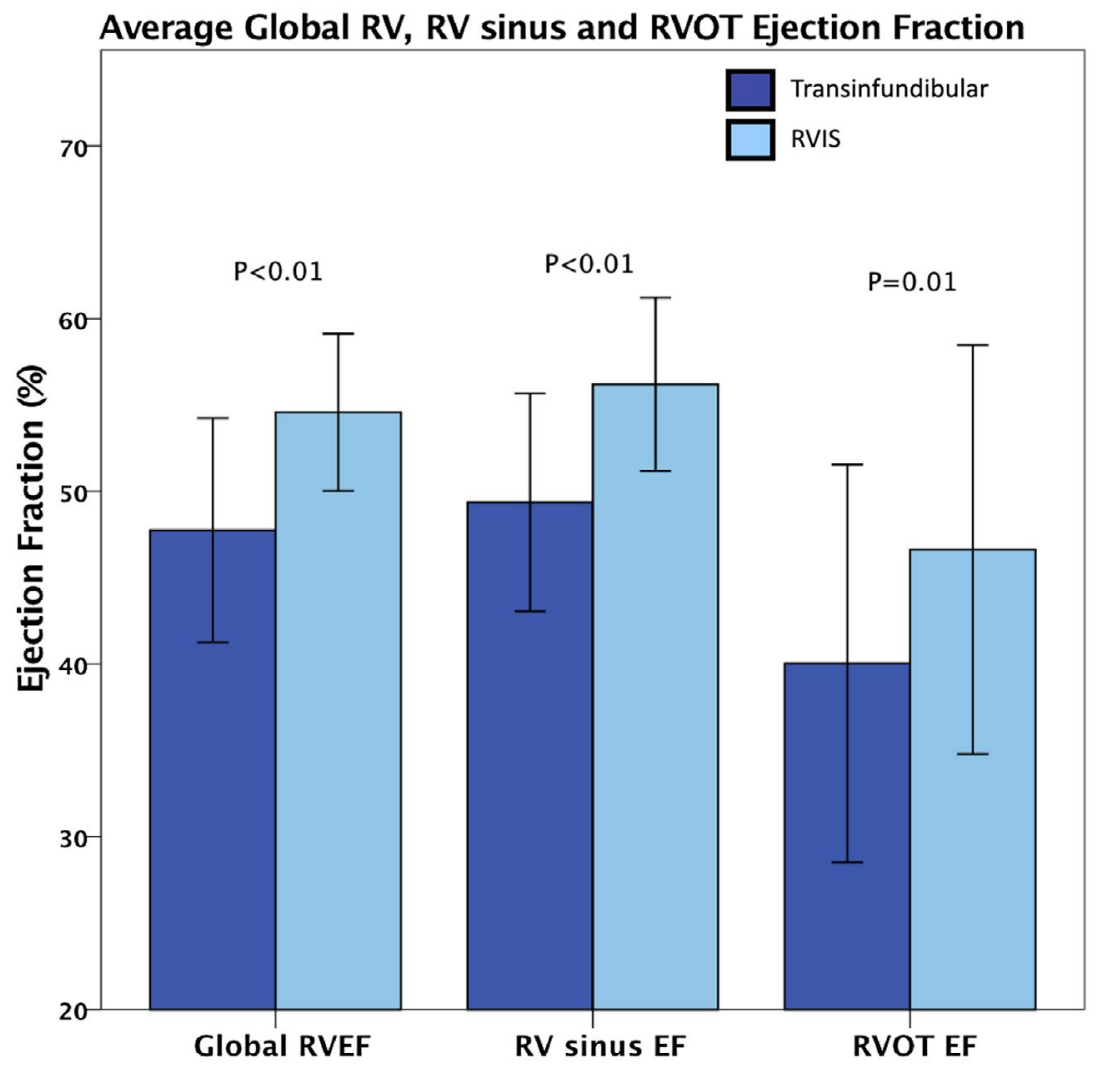

FIGURE 2 Average global RV ejection fraction, RVOT ejection fraction, and RV sinus EF for the transinfundibular (dark blue) and RVIS (light blue) groups. Error bars represent $1 \mathrm{SD}$

\section{Association between right and left ventricular ejection fraction}

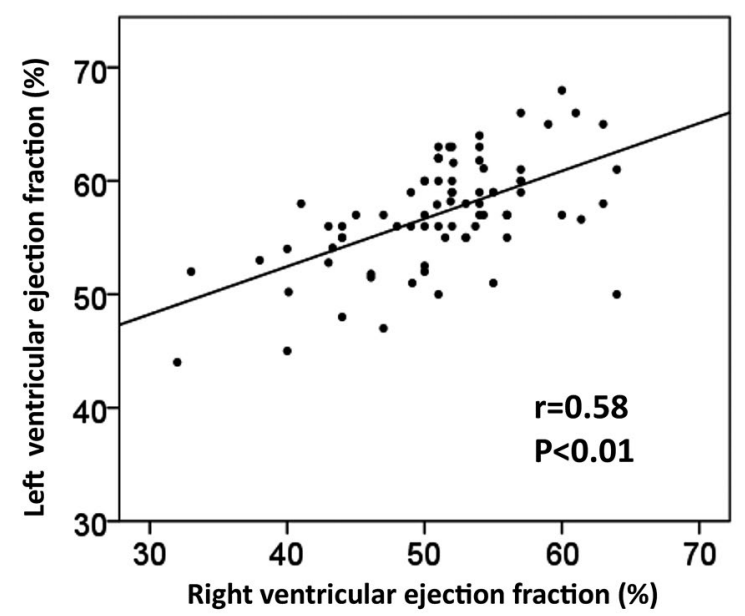

FIGURE 3 Association between left and right ventricular ejection fraction (entire cohort)

In a multivariable model that included surgical strategy (RVIS vs TI), age at CMR, and gender, female gender $(B=2.1, P=.11)$ and RVIS repair $(B=6.6, P<.005)$ were associated with higher RVEF with an adjusted $R^{2}=.28$. Female gender $(B=9.8, P=.07)$, younger age at CMR $(B=1.2, P=.03)$, and RVIS repair $(B=10.0$, $P=.03)$ were associated with lower RVESVi with an adjusted $R^{2}$ of .19. Younger age at CMR $(B=2.2, P=.01)$ and female gender ( $B=15.5, P=.08$ ) were associated with lower RVEDVi with an adjusted $R^{2}$ of .12 .

\section{4 | DISCUSSION}

We performed a retrospective cohort study comparing RV size and function between patients who underwent the RVIS with those who underwent TI. This represents the first direct comparison of CMR-based measurements of ventricular size and function in this setting. The RVIS cohort had higher average RVEF, an 8 fold reduction in odds of RV dysfunction, and lower average RVESVi than the TI group. RV outflow tract EF, RV sinus EF and LVEF were higher in the RVIS versus the TI group. Multivariable analyses revealed the RVIS approach to be associated with higher RVEF when accounting for female gender and age. Transannular incision was associated with higher PRF.

Prior studies have demonstrated a 25 year survival of $96.8 \%$ in patients who underwent transatrial/transpulmonary repair of TOF, with higher freedom from reoperation compared to classic repair, suggesting a lower incidence of pulmonary valve replacement. ${ }^{1}$ Recently, Lee et al identified TI incision of $\geq 1 \mathrm{~cm}$ an independent risk factor for RV dysfunction. ${ }^{20}$ The same group investigated whether patients with a "limited" ventriculotomy $(<1 \mathrm{~cm})$ had improved RV size and function compared to those who had a "conventional" ventriculotomy $(>1 \mathrm{~cm})$, and found no difference between the two groups. ${ }^{21}$ Importantly, our study compared patients who underwent any infundibulotomy ( $\mathrm{TI}$ ) to those in whom no infundibulotomy was performed (RVIS). Within this context, our findings suggest that the presence of even a "limited" $(1 \mathrm{~cm})$ infundibulotomy imparts a longterm maladaptive effect on RV function. 
FIGURE 4 Average right ventricular ejection fraction for the TI and RVIS groups within each age category. Error bars represent $1 \mathrm{SD}$
FIGURE 5 Average right ventricular end-diastolic and end-systolic volume for the TI (dark blue) and RVIS (light blue) groups. Error bars represent 1 SD

\section{Average Right Ventricular Ejection Fraction within Age Categories}

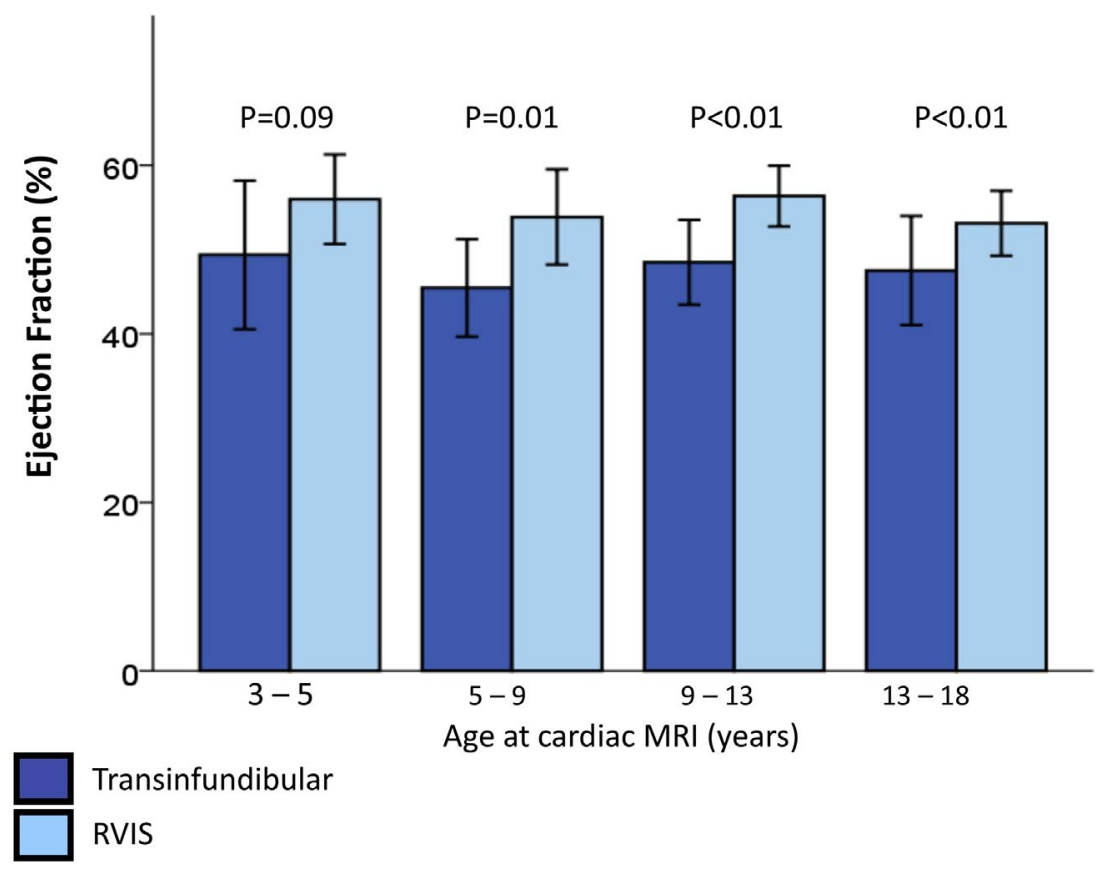

Average Right Ventricular End-Diastolic and End-Systolic Volumes
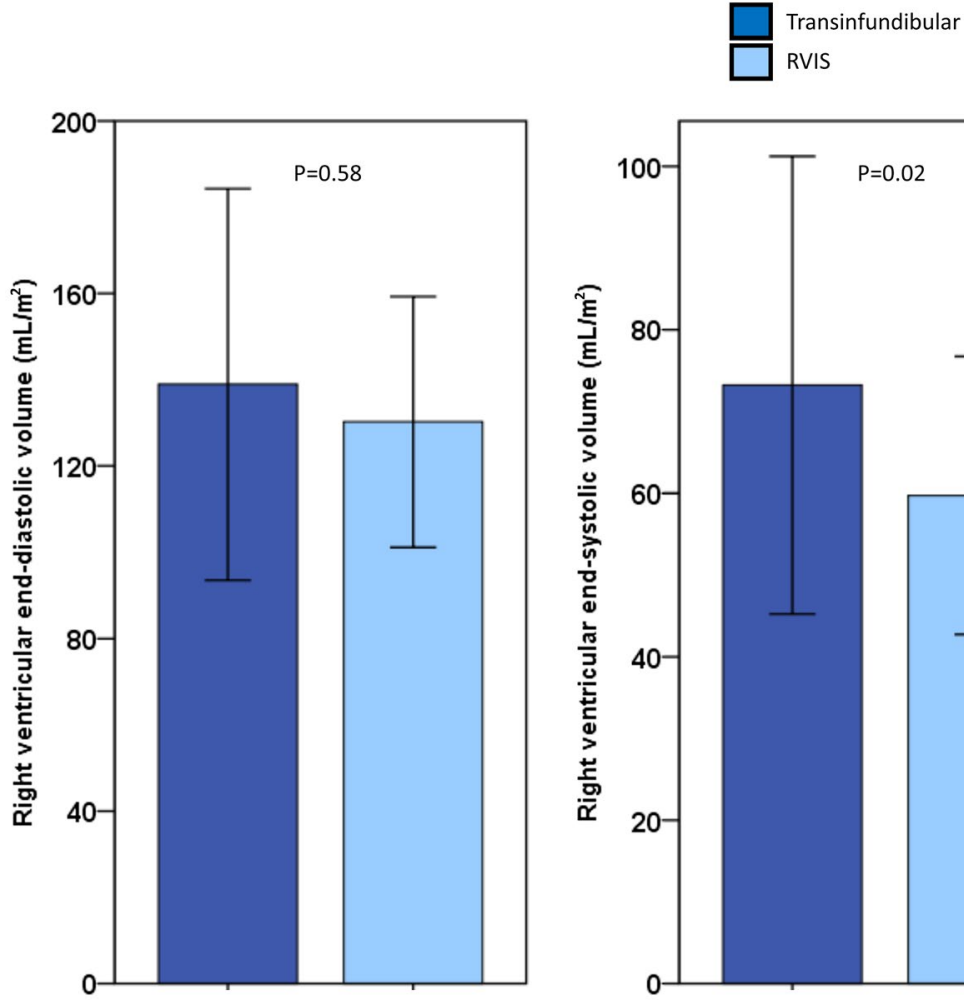

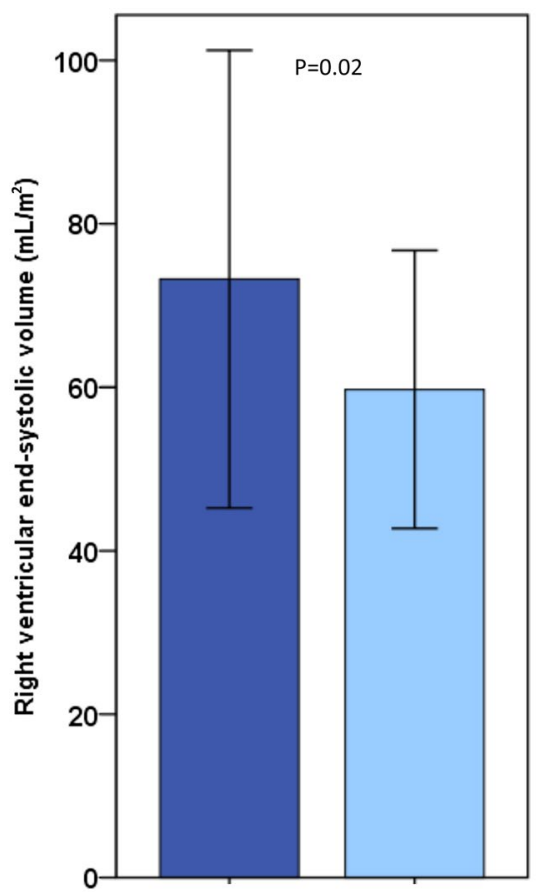

We speculate that the higher RVEF we observed in the RVIS group is due to preservation of a contractile infundibulum. In a porcine model, an infundibulotomy results in greater RV dilation and reduced RV function. ${ }^{9}$ In the setting of patients with TOF, delay in RVOT excursion correlates with QRS duration, suggesting that scarring of the RVOT leads to mechanical dyssynchrony, ${ }^{22}$ a known risk factor for RV dysfunction and sudden cardiac death. ${ }^{23} \mathrm{RV}$ outflow tract dysfunction correlates with overall RV dysfunction, LV dysfunction, and exercise capacity. ${ }^{24,25}$ Our findings of lower RVOT EF and global EF in the TI group are consistent with the hypothesis that early infundibular injury has lasting consequence. One unexpected finding was higher RV sinus EF in the RVIS group. The presence of an infundibular incision and subsequent scar may result in increased wall stress and subsequent adverse remodeling distal to the incision 
TA B LE 3 Endpoints Stratified by Surgical Strategy

\begin{tabular}{|c|c|c|c|}
\hline & Transinfundibular & RVIS & \\
\hline & $N=39$ & $N=40$ & $P$ value \\
\hline $\begin{array}{l}\text { Pulmonary regurgitant } \\
\text { fraction (\%) }\end{array}$ & $35 \pm 17$ & $41 \pm 13$ & .08 \\
\hline RVEDVi $\left(\mathrm{mL} / \mathrm{m}^{2}\right)$ & $139 \pm 45$ & $130 \pm 29$ & .32 \\
\hline RVESVi $\left(\mathrm{mL} / \mathrm{m}^{2}\right)$ & $73 \pm 28$ & $60 \pm 17$ & .01 \\
\hline RV Sinus EDVi $\left(\mathrm{mL} / \mathrm{m}^{2}\right)$ & $113 \pm 37$ & $105 \pm 24$ & .31 \\
\hline RV Sinus ESVi $\left(\mathrm{mL} / \mathrm{m}^{2}\right)$ & $57 \pm 22$ & $46 \pm 13$ & .01 \\
\hline RVOT EDVi (mL/m²) & $26 \pm 10$ & $25 \pm 8$ & .45 \\
\hline RVOT ESVi (mL/m²) & $16 \pm 8$ & $13 \pm 5$ & .08 \\
\hline LVEDVi $\left(\mathrm{mL} / \mathrm{m}^{2}\right)$ & $78 \pm 19$ & $71 \pm 13$ & .07 \\
\hline LVESVi $\left(\mathrm{mL} / \mathrm{m}^{2}\right)$ & $35 \pm 9$ & $29 \pm 7$ & $<.01$ \\
\hline
\end{tabular}

Notes: RVEDVi (indexed right ventricular end-diastolic volume), RVESVi (indexed right ventricular end-systolic volume), RV Sinus EDVi (indexed right ventricular sinus end-diastolic volume), RV Sinus ESVi (indexed right ventricular sinus end-systolic volume), RVOT EDVi (indexed right ventricular outflow tract end-diastolic volume), RVOT ESVi (indexed right ventricular outflow tract end-systolic volume), LVEDVi (indexed left ventricular end-diastolic volume), LVESVi (indexed left ventricular end-systolic volume), All volumes indexed to body surface area.

TABLE 4 Endpoints stratified by transannular patch

\begin{tabular}{|c|c|c|c|}
\hline & Transannular patch & No patch & \multirow[b]{2}{*}{$P$ value } \\
\hline & $(N=64)$ & $(N=15)$ & \\
\hline $\begin{array}{l}\text { Pulmonary regurgitant } \\
\text { fraction (\%) }\end{array}$ & $40 \pm 15$ & $30 \pm 15$ & .03 \\
\hline RVEDVi $\left(\mathrm{mL} / \mathrm{m}^{2}\right)$ & $134 \pm 39$ & $136 \pm 34$ & .85 \\
\hline RVESVi $\left(\mathrm{mL} / \mathrm{m}^{2}\right)$ & $66 \pm 24$ & $69 \pm 23$ & .60 \\
\hline RV Sinus EDVi $\left(\mathrm{mL} / \mathrm{m}^{2}\right)$ & $108 \pm 32$ & $111 \pm 26$ & .80 \\
\hline RV Sinus ESVi $\left(\mathrm{mL} / \mathrm{m}^{2}\right)$ & $51 \pm 19$ & $55 \pm 17$ & .54 \\
\hline RVOT EDVi $\left(\mathrm{mL} / \mathrm{m}^{2}\right)$ & $26 \pm 9$ & $25 \pm 10$ & .94 \\
\hline RVOT ESVi $\left(\mathrm{mL} / \mathrm{m}^{2}\right)$ & $15 \pm 7$ & $15 \pm 8$ & .88 \\
\hline LVEDVi $\left(\mathrm{mL} / \mathrm{m}^{2}\right)$ & $73 \pm 18$ & $80 \pm 12$ & .13 \\
\hline LVESVi $\left(\mathrm{mL} / \mathrm{m}^{2}\right)$ & $31 \pm 9$ & $35 \pm 7$ & .10 \\
\hline
\end{tabular}

Notes: RVEDVi (indexed right ventricular end-diastolic volume), RVESVi (indexed right ventricular end-systolic volume), RV Sinus EDVi (indexed right ventricular sinus end-diastolic volume), RV Sinus ESVi (indexed right ventricular sinus end-systolic volume), RVOT EDVi (indexed right ventricular outflow tract end-diastolic volume), RVOT ESVi (indexed right ventricular outflow tract end-systolic volume), LVEDVi (indexed left ventricular end-diastolic volume), LVESVi (indexed left ventricular end-systolic volume), All volumes indexed to body surface area.

itself. Alternatively, the TI incision in TI cases may have been extended inferior to where we defined the boundary of the RVOT.

There was no difference in RVEDVi and PRF between the RVIS and $\mathrm{TI}$ group, and this may be impacted by the inherent selection bias within the study design. The lower RVESVi in the RVIS group is not surprising, and may be indictive of improved compensatory mechanisms to volume overload in this cohort. The development of RV dysfunction is multifactorial, and, while chronic pulmonary regurgitation and $\mathrm{RV}$ volume overload are initially well tolerated, eventually compensentory mechanisms of the ventricle to volume overload fail, resulting in increased end-systolic volume and reduced $\mathrm{EF}^{19}$

There may be further hemodynamic effects of the RVIS approach that were not specifically elucidated during this study but which also lead to the observed difference in RVEF. While we did not assess the degree of residual obstruction in each group, no patient underwent repeat surgery for RV obstruction. It is possible that patients in the RVIS cohort had mild, subclinical RVOT obstruction which may impart a hemodynamic benefit in the setting of repaired TOF. $^{26}$ Patients who undergo RVIS are often treated with beta blockade in the postoperative period to protect against tachycardia which may impair RV filling and worsen RVOT obstruction. However, the degree of residual RVOT obstruction in patients who undergo the RVIS approach is rarely clinically significant in the long-term. ${ }^{12}$ While older age at repair may be seen as a disadvantage of the RVIS approach, the myocardium earlier in infancy may be more at risk for injury than later in infancy. Although later increase in RV mass as measured by CMR is a risk factor for poor clinical outcome in the setting of repaired TOF, RV hypertrophy has been correlated with improved contractility in an animal model. ${ }^{5,27}$ The correct degree of mild RV obstruction and hypertrophy may preserve contractility and limit maladaptive RV remodeling.

Other potential consequences of the RVIS approach may include surgical approaches that are altered in order to preserve the infundibulum. While there were no statistically significant differences in transannular incision and PRF between RVIS and TI groups, these analyses may suffer from lack of power. The authors believe that the presence of annular hypoplasia determines the need to perform a transannular incision. However, there may be differences in surgical approach related to the preservation of the infundibulum that have long-term consequence and are unaccounted for by our analyses.

Our finding of a higher LVEF in the RVIS group is likely secondary to favorable ventricular-ventricular interactions. Davlouros et al showed that RVOT akinesis resulted in decreased RVEF, and found a correlation between RVEF and LVEF, similar to our study. ${ }^{7,24}$ Transannluar incision did not appear to be associated with worsened RVOT EF, although it was associated with higher PRF. This supports the hypothesis that sparing the infundibulum and the pulmonary valve annulus are both important to long-term RV health, via different mechanisms. RV dysfunction and volume overload have been linked to long-term outcomes in patients with repaired TOF., ${ }^{4,7}$ The degree of volume overload may not be modified by the RVIS approach, preservation of the infundibulum appears mechanistically important for overall RV systolic function.

The impact of gender on outcomes in congenital heart disease has been difficult to establish due to the infrequency of cardiac lesions and heterogeneity within malformations. While data on children suggest that males are at higher risk for high risk congenital heart disease, females are at higher risk for mortality when high risk 
disease exists. ${ }^{28}$ Sarikouch et al reported higher RV end-systolic and end-diastolic volumes and lower RVEF in male patients with repaired TOF compared to females. However, when standardized to healthy controls, females actually demonstrated higher zscores for RV volumes and lower zscores for RVEF. ${ }^{8}$ Our findings of higher RVEDVi and lower RVEF in male patients may be reflective of the characteristic gender differences seen in the general population.

\section{5 | LIMITATIONS}

There are some inherent limitations of this study. As they reflect differences inherent to the approach, age at repair and the need for an intervening systemic to pulmonary artery shunt were not controlled for in the multivariable model. On univariate analysis, these factors did not appear to be associated with any of the outcome measures within each cohort. Early in the RVIS experience, repair was delayed until later in infancy. For this reason, the median age at TOF repair was 9.8 months. Other confounding differences in intraoperative and perioperative management may exist between and among the two institutions. While substantial differences exist between the centers in surgical volume, both are high volume centers with low surgical mortality. We did not analyze these other potential confounding factors, as the detail in the medical record did not allow for this. A certain degree of variability exists when measuring RV volumes, especially in the context of an immobile patch. We accounted for this by using a single, blinded, observer to measure $\mathrm{RV}$ volumes. Selection bias likely exists, with increased referral of patients for CMR in the presence of worsening RV dilation or dysfunction on echocardiogram, especially in younger age groups. The decision of when to provide pulmonary valve competency in the setting of chronic pulmonary insufficiency is somewhat subjective. These sources of bias may have contributed to the negative finding of differences in PRF and RVEDVi between the two cohorts and to the relatively high rates of transannular incision. Although we did not have sufficient data to match patients based on characteristics of their preoperative anatomy, patients with significant pulmonary valve hypoplasia who required a right ventricule to pulmonary artery conduit were excluded from the analysis. In addition, patients were enrolled based on the clinical indication to obtain a CMR, therefore the study population is likely biased toward patients with significant preoperative RV outflow tract obstruction. After study planning and data collection had begun, more recent data on risk factors for adverse events in TOF have identified RVEF cutoffs of $50 \%$ (female) and $48 \%$ (male). ${ }^{5}$ These values are slightly higher than the $45 \%$ cutoff value used in this study.

\section{6 | CONCLUSION}

In this study, the RVIS approach to repair of TOF was associated with higher RVEF, RVOT EF, RV sinus EF, and LVEF, lower RVESVi, and an 8 fold reduction in the odds of $\mathrm{RV}$ dysfunction compared to the
TI approach. These potential benefits must be balanced against the need to delay the TOF repair to 6-8 months of age and occasional need for a systemic to pulmonary artery shunt.

\section{CONFLICT OF INTEREST}

There are no conflicts of interest to disclose.

\section{AUTHOR CONTRIBUTIONS}

CONCEPT and design, data collection, drafting of the manusciprt, approval of the article: Olive

Concept and design, critical revision of the article, approval of article: Fraser

Data collection, concept and design, critical revision of the article, approval of the article: Kutty

Concept and design, critical revision of the article, approval of the article: Hammel, McKenzie

Concept and design, critical revision, approval of the article: Krishnamurthy

Data collection, critical revision, approval of the article: Dodd

Concept and design, critical revision of the article, statistics, approval of the article: Maskatia

\section{ORCID}

Mary K. Olive (iD https://orcid.org/0000-0001-6095-4701

Shiraz A. Maskatia (iD https://orcid.org/0000-0002-8806-1398

\section{REFERENCES}

1. d'Udekem Y, Galati JC, Rolley GJ, et al. Low risk of pulmonary valve implantation after a policy of transatrial repair of tetralogy of Fallot delayed beyond the neonatal period: the Melbourne experience over 25 years. J Am Coll Cardiol. 2014;63:563-568.

2. Kirsch RE, Glatz AC, Gaynor JW, et al. Results of elective repair at 6 months or younger in 277 patients with tetralogy of Fallot: a 14-year experience at a single center. J Thorac Cardiovasc Surg. 2014;147:713-717.

3. Geva T, Sandweiss BM, Gauvreau K, Lock JE, Powell AJ. Factors associated with impaired clinical status in long-term survivors of tetralogy of Fallot repair evaluated by magnetic resonance imaging. J Am Coll Cardiol. 2004;43:1068-1074.

4. Knauth AL, Gauvreau K, Powell AJ, et al. Ventricular size and function assessed by cardiac MRI predict major adverse clinical outcomes late after tetralogy of Fallot repair. Heart. 2008;94:211-216.

5. Valente AM, Gauvreau K, Assenza GE, et al. Contemporary predictors of death and sustained ventricular tachycardia in patients with repaired tetralogy of Fallot enrolled in the INDICATOR cohort. Heart. 2014;100:247-253.

6. Maskatia SA, Spinner JA, Nutting AC, Slesnick TC, Krishnamurthy $\mathrm{R}$, Morris SA. Impact of obesity on ventricular size and function in children, adolescents and adults with tetralogy of fallot after initial repair. Am J Cardiol. 2013;112(4):594-598.

7. Ortega M, Triedman JK, Geva T, Harrild DM. Relation of left ventricular dyssynchrony measured by cardiac magnetic resonance tissue tracking in repaired tetralogy of fallot to ventricular tachycardia and death. Am J Cardiol. 2011;107:1535-1540.

8. Sarikouch S, Boethig D, Peters B, et al. Poorer right ventricular systolic function and exercise capacity in women after repair of tetralogy of fallot: a sex comparison of standard deviation scores based on sex-specific reference values in healthy control subjects. Circ Cardiovasc Imaging. 2013;6:924-933. 
9. Bove T, Bouchez S, De Hert S, et al. Acute and chronic effects of dysfunction of right ventricular outflow tract components on right ventricular performance in a porcine model: implications for primary repair of tetralogy of fallot. J Am Coll Cardiol. 2012;60:64-71.

10. Bacha EA, Scheule AM, Zurakowski D, et al. Long-term results after early primary repair of tetralogy of Fallot. J Thorac Cardiovasc Surg. 2001;122:154-161.

11. Di Donato RM, Jonas RA, Lang P, Rome JJ, Mayer JE Jr, Castaneda AR. Neonatal repair of tetralogy of Fallot with and without pulmonary atresia. J Thorac Cardiovasc Surg. 1991;101:126-137.

12. Morales DL, Zafar F, Heinle JS, et al. Right ventricular infundibulum sparing (RVIS) tetralogy of fallot repair: a review of over 300 patients. Ann Surg. 2009;250:611-617.

13. McKenzie ED, Maskatia SA, Mery C. Surgical management of tetralogy of fallot: in defense of the infundibulum. Semin Thorac Cardiovasc Surg. 2013;25:206-212.

14. Chung T. Assessment of cardiovascular anatomy in patients with congenital heart disease by magnetic resonance imaging. Pediatr Cardiol. 2000;21:18-26.

15. O'Meagher S, Ganigara M, Munoz P, et al. Right ventricular outflow tract enlargement prior to pulmonary valve replacement is associated with poorer structural and functional outcomes, in adults with repaired Tetralogy of Fallot. Heart Lung Circ. 2014;23:482-488.

16. Bove $\mathrm{T}$, Vandekerckhove $\mathrm{K}$, Devos $\mathrm{D}$, et al. Functional analysis of the anatomical right ventricular components: should assessment of right ventricular function after repair of tetralogy of Fallot be refined? Eur J Cardiothorac Surg. 2014;45:e6-e12.

17. Bodhey NK, Beerbaum P, Sarikouch S, et al. Functional analysis of the components of the right ventricle in the setting of tetralogy of Fallot. Circ Cardiovasc Imaging. 2008;1:141-147.

18. Mercer-Rosa L, Yang W, Kutty S, Rychik J, Fogel M, Goldmuntz E. Quantifying pulmonary regurgitation and right ventricular function in surgically repaired tetralogy of Fallot: a comparative analysis of echocardiography and magnetic resonance imaging. Circ Cardiovasc Imaging. 2012;5:637-643.

19. Geva T. Repaired tetralogy of Fallot: the roles of cardiovascular magnetic resonance in evaluating pathophysiology and for pulmonary valve replacement decision support. J Cardiovasc Magn Reson. 2011;13:9.

20. Lee C, Lee $\mathrm{CH}$, Kwak JG, et al. Factors associated with right ventricular dilatation and dysfunction in patients with chronic pulmonary regurgitation after repair of tetralogy of Fallot: analysis of magnetic resonance imaging data from 218 patients. J Thorac Cardiovasc Surg. 2014;148:2589-2595.
21. Lee C, Lee $\mathrm{CH}$, Kwak JG, et al. Does limited right ventriculotomy prevent right ventricular dilatation and dysfunction in patients who undergo transannular repair of tetralogy of Fallot? Matched comparison of magnetic resonance imaging parameters with conventional right ventriculotomy long-term after repair. J Thorac Cardiovasc Surg. 2014;147:889-895.

22. Uebing A, Gibson DG, Babu-Narayan SV, et al. Right ventricular mechanics and QRS duration in patients with repaired tetralogy of Fallot: implications of infundibular disease. Circulation. 2007;116:1532-1539.

23. Gatzoulis MA, Balaji S, Webber SA, et al. Risk factors for arrhythmia and sudden cardiac death late after repair of tetralogy of Fallot: a multicentre study. Lancet. 2000;356:975-981.

24. Davlouros PA, Kilner PJ, Hornung TS, et al. Right ventricular function in adults with repaired tetralogy of Fallot assessed with cardiovascular magnetic resonance imaging: detrimental role of right ventricular outflow aneurysms or akinesia and adverse right-to-left ventricular interaction. J Am Coll Cardiol. 2002;40:2044-2052.

25. Wald RM, Haber I, Wald R, Valente AM, Powell AJ, Geva T. Effects of regional dysfunction and late gadolinium enhancement on global right ventricular function and exercise capacity in patients with repaired tetralogy of Fallot. Circulation. 2009;119:1370-1377.

26. Latus $\mathrm{H}$, Hachmann $\mathrm{P}$, Gummel $\mathrm{K}$, et al. Impact of residual right ventricular outflow tract obstruction on biventricular strain and synchrony in patients after repair of tetralogy of Fallot: a cardiac magnetic resonance feature tracking study. Eur J Cardiothorac Surg. 2015;48:83-90.

27. Bove T, Vandekerckhove K, Bouchez S, Wouters P, Somers P, Van Nooten G. Role of myocardial hypertrophy on acute and chronic right ventricular performance in relation to chronic volume overload in a porcine model: relevance for the surgical management of tetralogy of Fallot. J Thorac Cardiovasc Surg. 2014;147:1956-1965.

28. Marelli A, Gauvreau K, Landzberg M, Jenkins K. Sex differences in mortality in children undergoing congenital heart disease surgery: a United States population-based study. Circulation. 2010;122:S234-S240.

How to cite this article: Olive MK, Fraser CD, Kutty S, et al. Infundibular sparing versus transinfundibular approach to the repair of tetralogy of Fallot. Congenital Heart Disease.

2019;14:1149-1156. https://doi.org/10.1111/chd.12863 